\title{
Looking for hints of a reconstructible seesaw model at the Large Hadron Collider
}

\author{
Gulab Bambhaniya, ${ }^{1, *}$ Srubabati Goswami,, , + Subrata

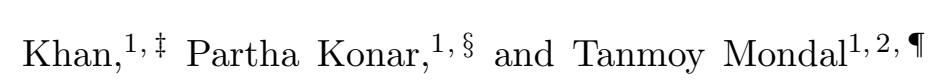 \\ ${ }^{1}$ Physical Research Laboratory (PRL), Ahmedabad-380009, Gujarat, India \\ ${ }^{2}$ Indian Institute of Technology, Gandhinagar, India.
}

(Dated: August 8, 2018)

\begin{abstract}
We study the production of heavy neutrinos at the Large Hadron Collider (LHC) through the dominant s-channel production mode as well as the vector boson fusion (VBF) process. We consider the TeV scale minimal linear seesaw model containing two heavy singlets with opposite lepton number. This model is fully reconstructible from oscillation data apart from an overall normalization constant which can be constrained from meta-stability of the electroweak vacuum and bounds coming from lepton flavor violation (LFV) searches. Dirac nature of heavy neutrinos in this model implies suppression of the conventional samesign-dilepton signal at the LHC. We analyze the collider signatures with tri-lepton final state and missing transverse energy as well as VBF type signals which are characterized by two additional forward tagged jets. Our investigation reveals that due to stringent constraints on light-heavy mixing coming from LFV and meta-stability bounds, the model can be explored only for light to moderate mass range of heavy neutrinos. We also note that in case of a positive signal, flavor counting of the final tri-lepton channel can give information about the mass hierarchy of the light neutrinos.

PACS numbers: 12.60.-i, 14.60.St, 13.35.Hb, 13.85.Qk
\end{abstract}

Keywords: Beyond Standard Model, Heavy Neutrinos, Hadronic Colliders, Lepton production

*Electronic address: gulab@prl.res.in

${ }^{\dagger}$ Electronic address: sruba@prl.res.in

${ }^{\ddagger}$ Electronic address: subrata@prl.res.in

${ }^{\S}$ Electronic address: konar@prl.res.in

๑Electronic address: tanmoym@prl.res.in 


\section{INTRODUCTION}

The discovery of the Higgs boson at the Large Hadron Collider both by ATLAS [1] and CMS [2] collaborations have put the Standard Model (SM) on a firm footing. However, no signal of physics beyond the Standard Model (BSM) has been found so far at the LHC. On the other hand, convincing indications of BSM physics have already emerged from the phenomenon of neutrino oscillation observed in terrestrial experiments. These results have conclusively established that neutrinos have non-zero mass and flavor mixing. Oscillation data together with the cosmological bound on sum of neutrino masses $\left(\Sigma m_{i}<0.23 \mathrm{eV}\right.$ including the PLANCK data [3] ) indicate that neutrino masses are much smaller as compared to the other fermions in the SM. Such small masses can be generated naturally by the seesaw mechanism. The origin of seesaw is the dimension 5 effective operator $\frac{c_{5}}{M} L L H H$, where $L(H)$ being the SM lepton(Higgs) doublet and $c_{5}$ is a dimensionless coupling, $M$ is the mass scale at which the effective operator gets generated [4]. Such operators arise by integrating out heavy fields added to the SM Lagrangian and they violate lepton number by two units. The smallness of neutrino mass in these models is related to the scale of lepton number violation which is required to be very high $\sim \mathcal{O}\left(10^{15} \mathrm{GeV}\right)$ to generate neutrino masses in the right ballpark. The most economical in terms of particle contents is the type-I seesaw in which heavy singlet right-handed neutrinos are added to the SM Lagrangian [5 9]. However, the natural seesaw scale is far beyond the reach of the LHC. To have signatures of seesaw models at the LHC, the heavy neutrino $(N)$ mass needs to be $\sim \mathcal{O}(\mathrm{TeV})$. However, if one lowers the scale of seesaw to $\mathrm{TeV}$ then it also requires much smaller neutrino Yukawa couplings $\left(\sim 10^{-6}\right)$ to obtain correct light neutrino masses. Such small Yukawa couplings lead to suppression of the production of the heavy neutrinos in natural TeV scale Type-I seesaw models. This leads to the question whether it is possible to achieve both the requirements simultaneously, i.e. having TeV scale heavy neutrinos along with large Yukawa coupling leading to large light-heavy mixing. Such possibilities can be realized in some specific mass textures [10 18]. Other options include models with higherdimensional operators arising due to exchange of new particles belonging to larger representations 19 27], radiative mass generations [28 35] etc. One of the most popular options to generate TeV scale seesaw is through the inverse seesaw models in which one includes additional singlet states. These models were first proposed in the context of E(6) Grand Unified Theories [36]. In these models the seesaw scale is decoupled from the scale of lepton number violation and the smallness of neutrino mass originates from the small lepton number violating terms in the Lagrangian.

In type-I Seesaw model the heavy and light neutrinos are both Majorana particles. It is well 
known that Majorana nature of neutrinos can be established by observing a positive signal in neutrino-less double beta decay experiments. It was noticed in [37], in the context of the LeftRight symmetric model that resonant production of $N$ and its subsequent decay giving same-sign di-lepton (SSDL) signal in colliders can also constitute evidence for Majorana nature of neutrinos. Given the importance of this signal, there have been several studies of this channel at the hadron colliders [38-43] including searches at the LHC [44]. Enhanced contribution from infrared tchannel, especially for heavier masses was proposed [45, 46] together with s-channel production.

The heavy neutrinos in inverse seesaw model are of pseudo-Dirac nature and in this case the SSDL signal is suppressed by the small lepton number violating coupling. For such models the heavy neutrinos are produced by the s-channel process along with a charged lepton. This neutrino further decays to a second lepton (of sign opposite to the first lepton to conserve lepton number) together with a W-boson. The W-boson can further decay leptonically to produce a lepton and a neutrino. Thus the final signal consists of tri-lepton and missing energy which is expected to have tiny contamination from standard model backgrounds. Detailed studies including the SM background in the context of pseudo-Dirac neutrinos have been done in [42, 47]. Similar studies in the context of Left-Right symmetric model, non-minimal supersymmetric inverse seesaw models and Type-III seesaw model have been performed in [48], [49] and [50] respectively. Experimental searches for multi-lepton signals have been carried out by the CMS collaboration using an integrated luminosity of $19.5 \mathrm{fb}^{-1}$ with center of mass energy $\sqrt{s}=8 \mathrm{TeV}$ at the LHC [51]. They considered at least three leptons in the final state using a search strategy not specific to any particular model.

In this work, we consider the minimal linear seesaw model (MLSM) studied in [52, 53] as an example of the $\mathrm{TeV}$ scale seesaw model. This is a variant of the inverse seesaw model but in this case the minimal scheme consists of adding just two heavy singlets with opposite lepton number as opposed to four heavy neutrinos in canonical minimal inverse seesaw models [54]. It was shown in 52] that the Yukawa couplings matrices for this model can be fully reconstructed in terms of the oscillation parameters apart from an overall normalization factor. It was further shown in [53] that this normalization constant can be constrained from consideration of the meta-stability of the electroweak vacuum and lepton flavor violation bounds. The heavy neutrinos in this model are of Dirac type and the SSDL signal is suppressed ${ }^{1}$. In the context of this model we consider two possible production channels for the heavy neutrinos resulting in two different classes of signals. The first one of this is the s-channel process to produce heavy Dirac neutrinos associated with a lepton and

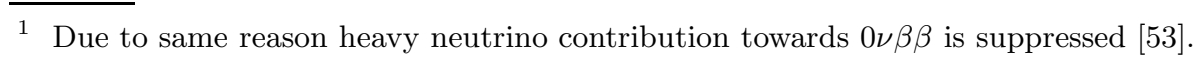


finally giving the tri-lepton and missing energy signal. The second one is the production of heavy neutrinos through vector boson fusion (VBF) in which two electroweak vector bosons coming from two partons 'fuse' to produce the signal under consideration (tri-leptons) along with two highly forward jets. It becomes important in the context of hadron colliders since the tagging of forward jets allows us to reduce the background considerably. Also the lack of color exchange between these jets makes the central region free from the color activities and this is exploited by vetoing central jets; see [55] and references therein in the context of Higgs search. This helps in minimizing the backgrounds further. For these reasons VBF remains an important channel to look for new physics [56-58] at hadron colliders.

We consider both normal hierarchy $(\mathrm{NH})$ as well as inverted hierarchy $(\mathrm{IH})$ for the light neutrino mass spectra. We also estimate the corresponding standard model backgrounds for the $14 \mathrm{TeV}$ LHC. In each case, we perform a realistic simulation with extensive event selections using MadGraph and PYTHIA.

The paper is organized as follows: Sec. 2 contains a brief description of the model. The production and decay of the right handed neutrino at LHC, are discussed in Sec. 3, Simulation details and results are presented in Sec. 4, while in Sec. 5 we discuss discovery potential of the signals at the LHC. Finally, we conclude in Sec. 6 ,

\section{THE LINEAR SEESAW MODEL}

The most general Lagrangian containing heavy singlet fields $N_{R}$ and $\mathrm{S}$ with opposite lepton numbers, is given by

$$
-\mathcal{L}=\bar{N}_{R} Y_{\nu} \tilde{\phi}^{\dagger} l_{L}+\bar{S} Y_{S} \tilde{\phi}^{\dagger} l_{L}+\bar{S} M_{N} N_{R}^{c}+\frac{1}{2} \bar{S} \mu S^{c}+\frac{1}{2} \overline{N_{R}} \mu_{N} N_{R}^{c}+\text { h.c. }
$$

where $l_{L}=\left(\nu_{x}, x\right)_{L}^{T}, x=e, \mu, \tau$.

Once the symmetry is broken spontaneously, the Higgs field $\phi$ obtains a vacuum expectation value $(\mathrm{VEV})$ equal to $v / \sqrt{2}$. This generates the Dirac mass term $m_{D}=Y_{\nu} v / \sqrt{2}$ and the lepton

number breaking mass term $m_{S}=Y_{S} v / \sqrt{2}$. In the linear seesaw models [59 61] one assumes $m_{S}$ to be small and non-zero while the $\mu$ and the $\mu_{N}$ terms are set to zero. This can be done since they contribute towards light neutrino mass in the sub-leading orders [62]. Since lepton number violating mass terms are set to zero, the heavy neutrinos are purely Dirac type. Then the mass 
matrix takes the form

$$
\mathcal{M}_{\nu}=\left(\begin{array}{ccc}
0 & m_{D}^{T} & m_{S}^{T} \\
m_{D} & 0 & M_{N} \\
m_{S} & M_{N}^{T} & 0
\end{array}\right)
$$

in the $\left(\nu_{L}, N_{R}^{c}, S^{c}\right)$ basis.

The minimal model which can successfully generate two light neutrinos with non-zero mass is when only two extra heavy singlets are added to the SM Lagrangian. This is called the Minimal Linear Seesaw Model (MLSM) [52, 53]. The full mass matrix has dimension $5 \times 5$ and can be written as ,

$$
\mathcal{M}_{\nu}=\left(\begin{array}{cc}
0 & m_{D}^{\prime T} \\
m_{D}^{\prime} & M
\end{array}\right)
$$

where $m_{D}^{T}=\left(m_{D}^{T}, m_{S}^{T}\right)$ and

$$
M=\left(\begin{array}{cc}
0 & M_{N} \\
M_{N} & 0
\end{array}\right) .
$$

For the minimal case $M_{N}$ is just a number, not a matrix. $\mathcal{M}_{\nu}$ can be diagonalized by a $5 \times 5$ unitary matrix $U_{0}$ as

$$
U_{0}^{T} \mathcal{M}_{\nu} U_{0}=\mathcal{M}_{\nu}^{\mathrm{d} i a g}
$$

where $\mathcal{M}_{\nu}^{\text {diag }}=\operatorname{diag}\left(m_{1}, m_{2}, m_{3}, M_{1}, M_{2}\right)$. Following a two-step diagonalization procedure [63], $U_{0}$ can be expressed as,

$$
U_{0}=\left(\begin{array}{cc}
\left(1-\frac{1}{2} \epsilon\right) U_{\nu} & m_{D}^{\dagger}\left(M^{-1}\right)^{*} U_{R} \\
-M^{-1} m_{D} U_{\nu} & \left(1-\frac{1}{2} \epsilon^{\prime}\right) U_{R}
\end{array}\right) \equiv\left(\begin{array}{cc}
U_{L} & V \\
S & U_{H}
\end{array}\right),
$$

where, $U_{L}$ is the $U_{P M N S}$ mixing matrix, and $V, S$ are the light-heavy mixing matrices. Interaction of heavy neutrinos with the SM fields are determined by the mixing matrix V, whose elements will be denoted as $V_{l N}$ hereafter. We would notice afterwards that the strong constraints on some elements of this matrix i.e. $V_{e N}$ and $V_{\mu N}$ would restrict the production signal. The diagonalizing matrix is now non-unitary which is characterized by the factor $(1-\epsilon / 2)$. The non-unitary corrections $\epsilon$ and $\epsilon^{\prime}$ are given in [63, 64]. $U_{\nu}$ is the unitary component of $U_{P M N S}$ which is same as $U_{P M N S}$ for $\epsilon<<1$. We use the standard parametrization for this:

$$
U_{\nu}=\left(\begin{array}{ccc}
c_{12} c_{13} & s_{12} c_{13} & s_{13} e^{-i \delta} \\
-c_{23} s_{12}-s_{23} s_{13} c_{12} e^{i \delta} & c_{23} c_{12}-s_{23} s_{13} s_{12} e^{i \delta} & s_{23} c_{13} \\
s_{23} s_{12}-c_{23} s_{13} c_{12} e^{i \delta} & -s_{23} c_{12}-c_{23} s_{13} s_{12} e^{i \delta} & c_{23} c_{13}
\end{array}\right) P
$$


where $c_{i j}=\cos \theta_{i j}, s_{i j}=\sin \theta_{i j}$ and $\delta$ denotes the Dirac CP phase. The Majorana phase matrix $P$ is expressed as $P=\operatorname{diag}\left(e^{-i \alpha}, e^{i \alpha}, 1\right)$, there is only one Majorana phase because one of the mass eigenvalues is zero. In table I, we have presented the $3 \sigma$ allowed range of oscillation parameters. Note that the phases are completely unconstrained at present.

Using the seesaw approximation one obtains the light neutrino mass matrix,

$$
m_{\text {light }}=m_{D}^{\prime T} M^{-1} m_{D}^{\prime}
$$

This being a rank 2 matrix the light neutrinos belonging to this model are hierarchical. Thus there are two possible mass spectra:

$\diamond$ Normal Hierarchy $(\mathrm{NH}): \quad\left(m_{1}<m_{2}<m_{3}\right)$

$\diamond$ Inverted Hierarchy $(\mathrm{IH}): \quad\left(m_{3}<<m_{2} \approx m_{1}\right)$.

In MLSM, $Y_{\nu}$ and $Y_{S}$ are $3 \times 1$ matrices (cf. Eq. 1) and can be considered as two independent vectors

$$
Y_{\nu} \equiv y_{\nu} \hat{\mathbf{a}} ; \quad Y_{S} \equiv y_{s} \hat{\mathbf{b}}
$$

where $\hat{\mathbf{a}}$ and $\hat{\mathbf{b}}$ denotes complex vectors with unit norm while $y_{\nu}$ and $y_{s}$ represent the norms of the Yukawa matrices $Y_{\nu}$ and $Y_{S}$, respectively. Using Eq. 8 and 9 one can reconstruct the Yukawa matrices $Y_{\nu}$ and $Y_{S}$ in terms of the oscillation parameters barring an overall normalization factor. The parametrization of the Yukawa matrices depend on the mass hierarchy and can be expressed as $[52,53]$,

$$
\begin{aligned}
& Y_{\nu}=\frac{y_{\nu}}{\sqrt{2}}\left(\sqrt{1+\rho} U_{j}^{\dagger}+e^{i \frac{\pi}{2}} \sqrt{1-\rho} U_{k}^{\dagger}\right), \\
& Y_{S}=\frac{y_{s}}{\sqrt{2}}\left(\sqrt{1+\rho} U_{j}^{\dagger}-e^{i \frac{\pi}{2}} \sqrt{1-\rho} U_{k}^{\dagger}\right),
\end{aligned}
$$

where, $j=2, k=3$ for $\mathrm{NH}$ and $j=2, k=1$ for IH. $U_{j}$ 's denote the columns of the unitary matrix $U_{\nu}$ that diagonalizes the light neutrino mass matrix $m_{\text {light }}$ in Eq. 8. The parameter $\rho$ is given as,

$$
\rho=\frac{\sqrt{1+r}-\sqrt{r}}{\sqrt{1+r}+\sqrt{r}}(N H), \quad \rho=\frac{\sqrt{1+r}-1}{\sqrt{1+r}+1}(I H) .
$$

Here $r$ denotes the ratio of the solar and atmospheric mass squared differences, $r=\Delta m_{\odot}^{2} / \Delta m_{\text {atm }}^{2}$, with $\Delta m_{\odot}^{2} \equiv m_{2}^{2}-m_{1}^{2}$ and $\Delta m_{\text {atm }}^{2} \simeq m_{3}^{2}-m_{1}^{2} \quad\left(m_{2}^{2}-m_{3}^{2}\right)$ for $\mathrm{NH}(\mathrm{IH})$.

The overall coupling $y_{\nu}$ can be constrained from the metastability of the electro-weak vacuum and LFV [53]. For NH the most stringent constraint comes from LFV, whereas for IH case vacuum 
meta-stability constraint is more restrictive. This is because of cancellations occurring for IH for LFV processes [53]. The dependence of the bound on $y_{\nu}$ from meta-stability and LFV on the heavy neutrino mass has been shown in [53]. The metastability bound on $y_{\nu}$ varies approximately in the range $0.4-0.5$ for $M_{N}$ varying in the range $100-1000 \mathrm{GeV}$. This bound is indpendent of the oscillation parameters. However, significant variation on the bound on $y_{\nu}$ from LFV constraint is possible within the allowed range of oscillation parameters, mostly due to unconstrained phases, $\delta$ and $\alpha$. Details of these dependence can be followed from Fig. 1, For a particular $M_{N}$, the strength of the signal at LHC would depend on the value of $y_{\nu}$. To maximise the signal we therefore choose the value of $y_{\nu}$ at the peak for NH case. However, for IH case the peak value is much above the vacuum metastability bound and therefore we choose maximum allowed value of $y_{\nu}$ satisfying the metastability bound. The corresponding parameter values are depicted in table \ for NH (case I) and IH. Note that, the above mentioned cancellations within the terms, ensure the peak position corresponds to $\alpha+\delta=3 \pi / 2(3 \pi / 4)$ for $\mathrm{NH}(\mathrm{IH})$, which is also evident in Fig. 1. We have chosen $\delta=0$ in our analysis. For some other values of $\delta$, the phase $\alpha$ has to be chosen so that one is at the peak. In Fig. 1 we also show the variation of this bound with respect to the $\theta_{23}$ mixing angle in lower octant $\left(\mathrm{LO}, \theta_{23}<\pi / 4\right)$ and higher octant $\left(\mathrm{HO}, \theta_{23}>\pi / 4\right)$. The $y_{\nu}$ value $0.4(0.075)$ corresponds to $\mathrm{IH}\left(\mathrm{NH}\right.$ : Case-I) scenario for $M_{N}=100 \mathrm{GeV}$, which we will use in our analysis. These will be translated into the bounds on the mixing matrix elements, $V_{l N}$, depending on the heavy neutrino mass $M_{N}$. Since $y_{s}$ is extremely small $\left(\mathcal{O}\left(10^{-10}\right)\right), Y_{S}$ does not play any role in determining $V_{l N}$. The elements of the matrix $V\left(\right.$ or $\left.V_{l N}\right)$ can be expressed in terms of $U_{P M N S}$ matrix, $\rho$ and $y_{\nu}$ as follows:

$$
\begin{aligned}
V_{e N_{1}} & =\frac{-i}{\sqrt{2} M_{N}} \frac{y_{\nu} v}{2}\left[\sqrt{1+\rho}\left(U_{P M N S}\right)_{12}^{*}+i \sqrt{1-\rho}\left(U_{P M N S}\right)_{11}^{*}\right] \\
& \simeq \frac{y_{\nu} v}{4 M_{N}}\left[e^{i(\alpha+\delta)}(-2+\sqrt{r}) r^{\frac{1}{4}} s_{12}-2 i s_{13}\right]+\mathcal{O}\left(\left(\sqrt{r}, s_{13}\right)^{2}\right) \\
V_{\mu N_{1}} & =\frac{-i}{\sqrt{2} M_{N}} \frac{y_{\nu} v}{2}\left(\sqrt{1+\rho}\left(U_{P M N S}\right)_{22}^{*}+i \sqrt{1-\rho}\left(U_{P M N S}\right)_{21}^{*}\right) \\
& \simeq \frac{y_{\nu} v}{4 M_{N}}\left[(-2+\sqrt{r})\left(e^{i \alpha} r^{\frac{1}{4}} c_{12} c_{23}+i s_{23}\right)+2 e^{i(\alpha+\delta)} r^{\frac{1}{4}} s_{12} s_{23} s_{13}\right]+\mathcal{O}\left(\left(\sqrt{r}, s_{13}\right)^{2}\right) .
\end{aligned}
$$

The above expressions are for NH scenario and similar expressions can be computed for IH also. The element $V_{e N_{2}}\left(V_{\mu N_{2}}\right)$ differs from $V_{e N_{1}}\left(V_{\mu N_{1}}\right)$ by a phase factor. Note that in Table II, we also consider a second set of oscillation parameters for $\mathrm{NH}$ (NH: Case II) corresponding to a lower value of $y_{\nu}$ of 0.056 with $\theta_{23}$ in the higher octant. This value is chosen such that $V_{\mu N}$ is maximum and muon signal may be larger, since muon has higher efficiency for detection.

To get some perspective on the degree of suppression in cross section coming from these con- 

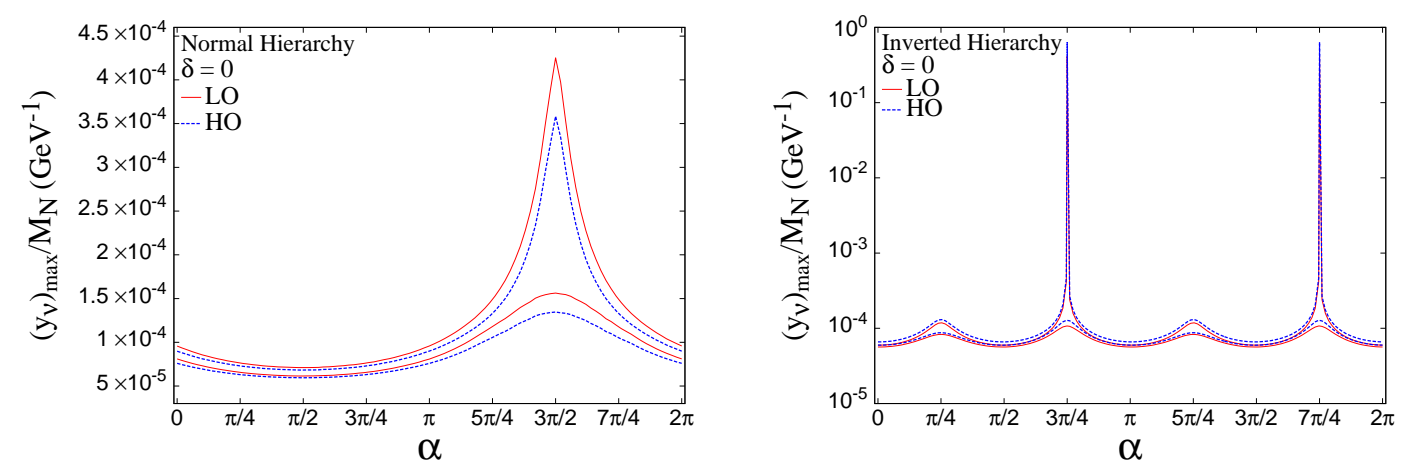

FIG. 1: Bound on $y_{\nu} / M_{N}$ as a function of Majorana phase $\alpha$, varying the oscillation parameters in the allowed $3 \sigma$ range. Red-solid(Blue-dashed) curve corresponds to atmoshpheric angle $\left(\theta_{23}\right)$ residing in $\mathrm{LO}(\mathrm{HO})$ region. (Left plot) The plot is for NH scenario, where highest allowed value of $y_{\nu} / M_{N}$ lies in LO region. (Right plot) The same plot for IH scenario.

\begin{tabular}{|c|c|c|c|c|c|c|}
\hline Bound $\quad$ Parameter & $\Delta_{\odot}^{2}\left[10^{-5} \mathrm{eV}^{2}\right]$ & $\Delta_{\mathrm{atm}}^{2}\left[10^{-3} \mathrm{eV}^{2}\right]$ & $\sin ^{2} \theta_{12}$ & $\sin ^{2} \theta_{23}$ & $\sin ^{2} \theta_{13}$ & $\delta$ \\
\hline $3 \sigma$ range $(\mathrm{NH})$ & $7.12-8.20$ & $2.31-2.74$ & $0.27-0.37$ & $0.36-0.68$ & $0.017-0.033$ & $0-2 \pi$ \\
\hline$(\mathrm{IH})$ & & $2.21-2.64$ & & $0.37-0.67$ & & \\
\hline Used value (NH: Case - I) & 7.15 & 2.73 & 0.27 & 0.36 & 0.033 & 0.0 \\
\hline Used value (NH: Case - II) & 7.13 & 2.73 & 0.27 & 0.68 & 0.033 & 0.0 \\
\hline Used value (IH) & 7.25 & 2.40 & 0.34 & 0.57 & 0.021 & 0.0 \\
\hline
\end{tabular}

TABLE I: Allowed $3 \sigma$ ranges of oscillation parameters and benchmark values of these parameters used in our analysis to get the signal allowed by LFV and vacuum metastability. Case-I corresponds to the peak in Fig. 1(Left panel), while Case-II corresponds to a lower value of $y_{\nu} / M_{N}$, for which $V_{\mu N}$ is maximum. Value of Majorana phase $\alpha$ is set at $3 \pi / 2(3 \pi / 4)$ for $\mathrm{NH}(\mathrm{IH})$ scenario.

straints we note down the corresponding $V_{l N}$ values for $M_{N}=100 \mathrm{GeV}$ as: $V_{e N}=1.95 \times 10^{-3}$, $V_{\mu N}=2.93 \times 10^{-2}$ and $V_{\tau N}=8.83 \times 10^{-2}$ for NH (Case-I) scenario, whereas, $V_{e N}=1.43 \times 10^{-3}$, $V_{\mu N}=4.14 \times 10^{-2}$ and $V_{\tau N}=5.48 \times 10^{-2}$ for $\mathrm{NH}$ (Case-II) respectively. For IH these values are $V_{e N}=0.48, V_{\mu N}=4.15 \times 10^{-9}$ and $V_{\tau N}=0.109$. Note that since our model is fully reconstructible and the only unknown parameter is $y_{\nu}$ which can be constrained from LFV and meta-stabilty bounds, we have definite predictions for the parameters $V_{l N}$ and these values are different for $\mathrm{NH}$ and IH scenarios. Bounds on $V_{l N}$ can also come from Electroweak Precision Data (EWPD) [65]. Our bounds for $\mathrm{NH}$ are consistent with these bounds. For IH we get a larger value for $V_{e N}$. However it is to be noted that the EWPD bounds are obtained assuming mixing with a single charged lepton and can be evaded in presence of cancellations or mixing with the other charged leptons 


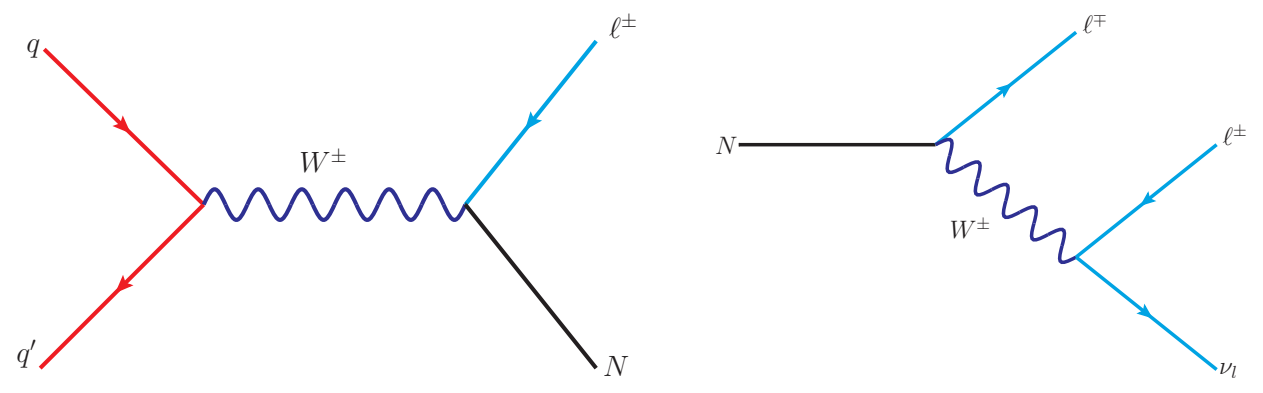

FIG. 2: (Left plot) Leading order s-channel diagram for heavy neutrino production at hadron colliders, and (Right plot) representative diagram for one of the decay mode of the heavy neutrino. These two figures lead to tri-lepton $+\mathbb{E}_{T}$ signal considered in the analysis.
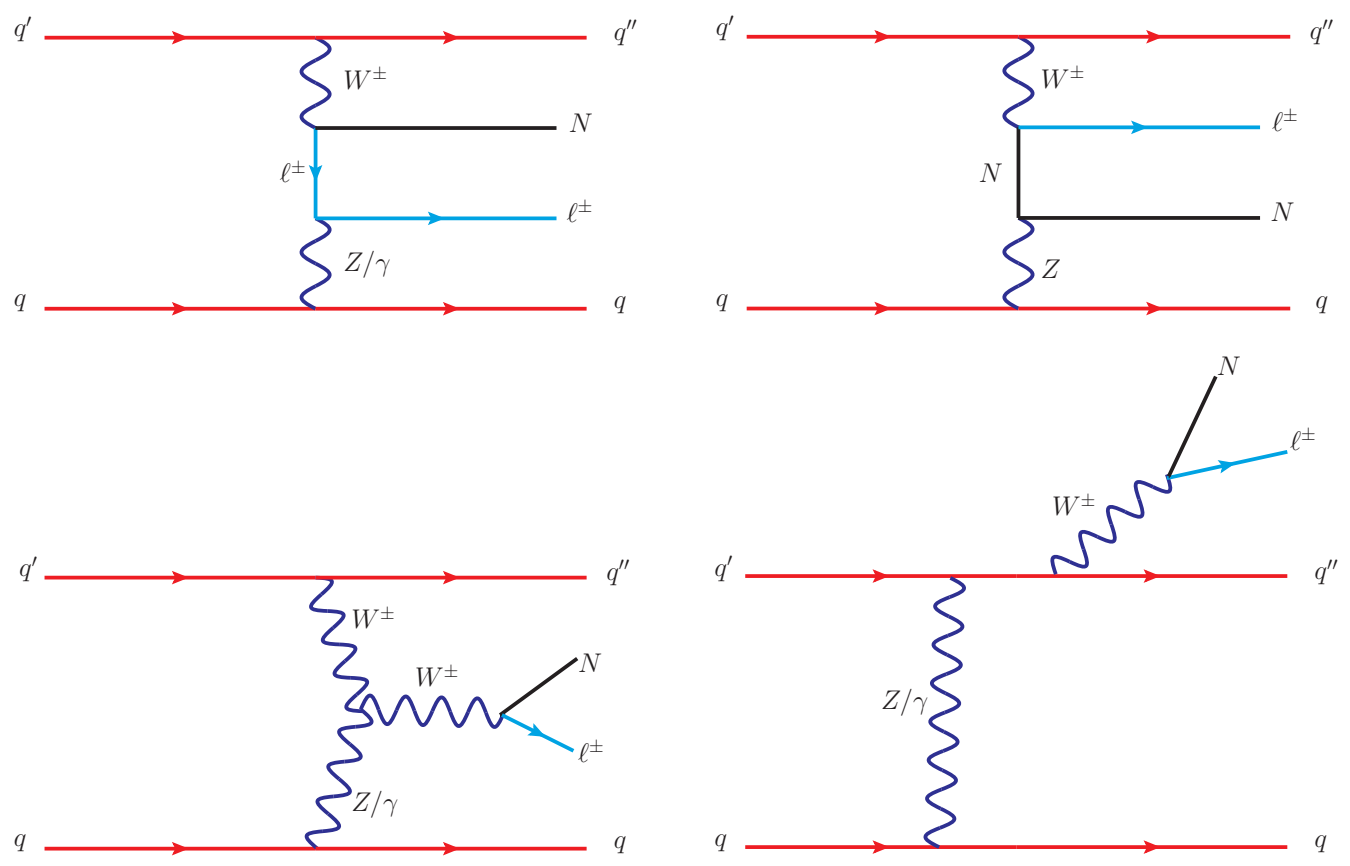

FIG. 3: Representative parton level diagrams contributing to $N \ell j j$ production through vector boson fusion at hadron colliders. Mirror diagrams are not shown here and also the last diagram is one of the four diagrams with $W^{ \pm}$emitting from each of the quark legs.

[42].

\section{PHENOMENOLOGY AT THE LHC}

The dominant production channel of the heavy neutrinos at LHC is the s-channel process through virtual W-boson exchange. At the leading order the parton level process $\left(q \overline{q^{\prime}} \rightarrow W^{ \pm} \rightarrow\right.$ $\left.\ell^{ \pm} N\right)$ is depicted in Fig. 2(left plot). The heavy neutrinos can also be produced through the 


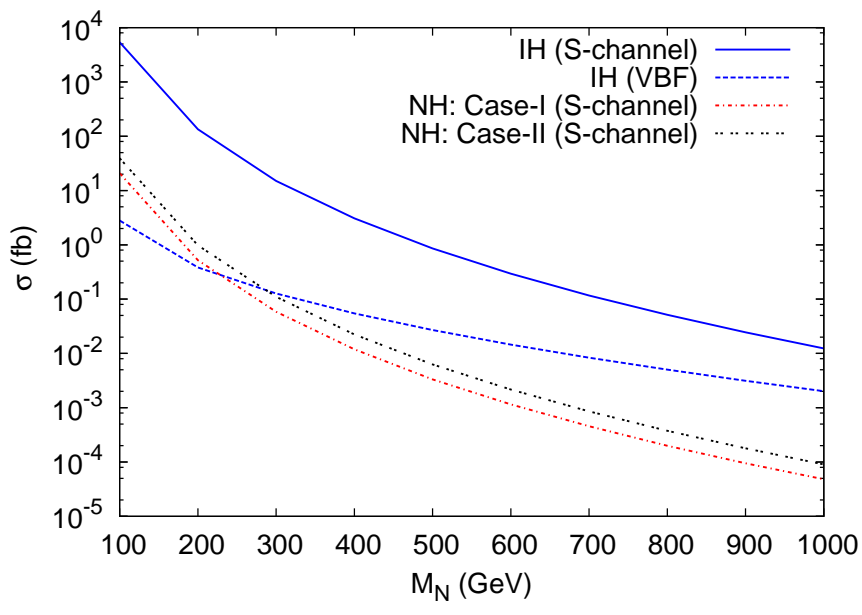

FIG. 4: The total cross section is shown for production of heavy neutrino associated with light lepton $(p p \rightarrow N \ell$, where $\ell=e, \mu)$ at the $14 \mathrm{TeV}$ LHC through the leading order s-channel process, while dotted lines represent VBF production cross section.

VBF process where production of $N$ is associated with two forward jets. Fig. 3 contains the representative parton level Feynman diagrams for VBF processes ${ }^{2}$. Estimated total production cross sections of these heavy Dirac neutrinos at the $14 \mathrm{TeV}$ LHC in IH scenario are shown in Fig. 4 for both $s$-channel(solid-line) as well as VBF (dashed-line). For NH scenario the $s$-channel production crossections are shown in the same figure for two different cases (c.f. Table I), Case-I ( Red dot-dashed line) and Case-II ( Black double dotted line). Basic cuts such as $p_{T \ell}>20 \mathrm{GeV}$ and $\left|\eta_{\ell}\right|<2.5$ are applied and $y_{\nu}$ values mentioned in the previous section are used. It is seen from the figure that although case II corresponds to a lower value of $y_{\nu}$ since $V_{\mu N}$ is larger, the production cross-section is slightly larger. Since the VBF cross-section is much lower we do not present the VBF cross-section for the NH case. In these analyses CTEQ6L1 [68] parton distribution functions have been used with the factorization scale set at the heavy neutrino mass $M_{N}$.

Heavy neutrinos $N$ can decay into charged lepton or neutrino associated with gauge (or Higgs) boson.

$$
N \rightarrow W^{ \pm} l^{\mp} / Z \nu_{l} / H \nu_{l}, \quad \text { where } l \equiv e, \mu, \tau .
$$

A representative diagram for decay of $N\left(N \rightarrow \ell^{\mp} W^{ \pm}\right)$is shown in Fig. 2(right plot).

In Fig. 5 we present the branching ratios for these decay channels as a function of heavy neutrino mass $M_{N}$ both in the case of normal hierarchy (left) and inverted hierarchy (right). Total decay

\footnotetext{
${ }^{2}$ Note that there are some diagrams which are not truly VBF type, i.e. two gauge boson are not fused via t-channel (e.g. bottom right diagram in Fig. 3), but they can lead to the same final states. These diagrams are necessary for the requirements of gauge invariance and included both for BG [66, 67] and signal calculations.
} 

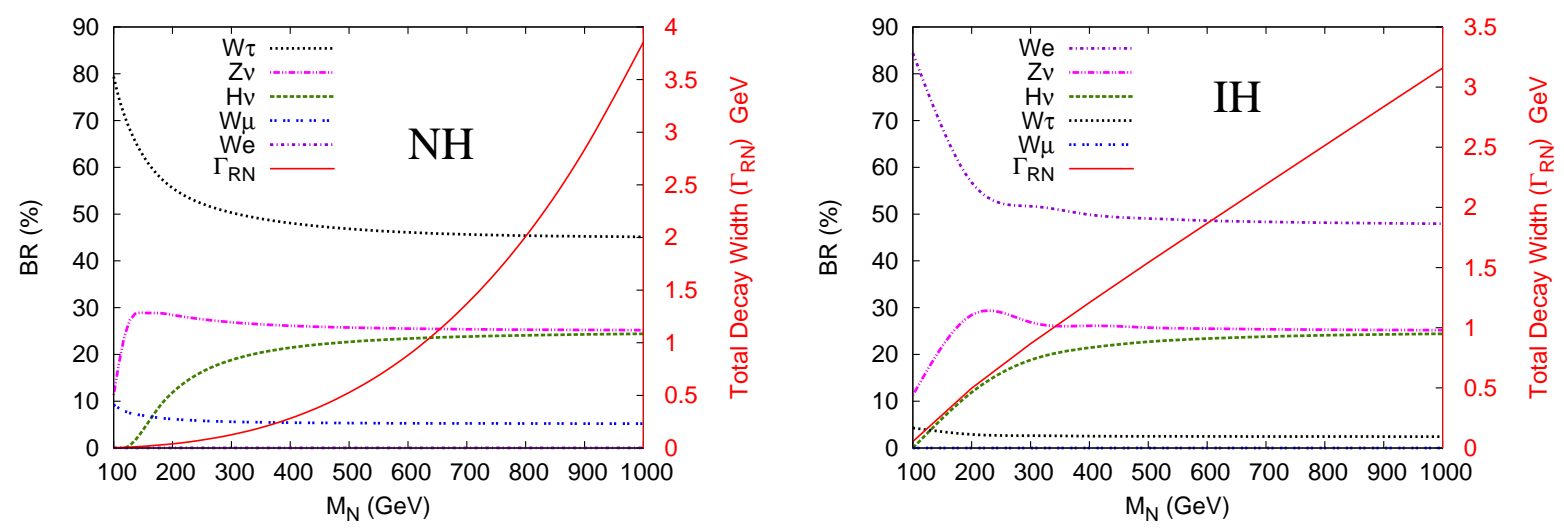

FIG. 5: The decay branching ratios of the heavy neutrino $(N)$ in different channels as a function of its mass in the case of normal hierarchy, Case-I, (left) and inverted hierarchy (right). Total decay widths in each case are also demonstrated with the solid line in the same figure.

widths in each case are also demonstrated with the solid line in each figure. Identifying that the charged lepton decay modes for heavy neutrino i.e. $N \rightarrow W^{ \pm} l^{\mp}$ being the main channel for search at the hadron collider, we discuss the corresponding decay modes in detail for both scenarios. The figure clearly shows that for NH, Case- $\mathrm{I}^{3}$, heavy neutrinos mostly decay into tau lepton $(\tau)$ and $W$ boson. On the other hand for IH, decay into the first generation lepton (e) possesses the maximum branching ratio. For $\mathrm{NH}$ the decay to $\mu$ is low and decay to $e$ is severely suppressed, while for $\mathrm{IH}$, the decay to $\tau$ has a lower ratio and decay to $\mu$ is negligible. The $W^{ \pm}$can have hadronic decay modes $\left(W^{ \pm} \rightarrow j j\right)$ or leptonic decay modes $\left(W^{ \pm} \rightarrow l^{ \pm} \nu\right)$. The tri-lepton signal $p p \rightarrow l^{ \pm} l^{\mp} l^{ \pm} \nu$ comes from the later decay mode ${ }^{4}$.

Other than charged lepton decay mode, $N$ can also decay to Z-boson or Higgs boson associated with neutrinos as listed in Eq. 13. The corresponding branching ratios are also shown in Fig. 5. Note that the branching ratio for $Z \nu$ is suppressed for lower values of the masses of the heavy neutrinos essentially because of $\mathrm{W}$ mass threshold. For the $H \nu$ decay mode, the Higgs mass threshold suppresses the decay rate for lower values of $M_{N} \sim 100 \mathrm{GeV}$. However, as $M_{N}$ increases these branching ratios increase to retain a $\sim 25 \%$ level. Both these channels can contribute to the

\footnotetext{
${ }^{3}$ For Case-II, although BRs to different channels likely to change, we do not show the corresponding plot as final production cross-section for both the cases, after putting all the selection criteria, is very low for NH and beyond the reach of LHC at $14 \mathrm{TeV}$ even with a luminosity of $3000 \mathrm{fb}^{-1}$.

${ }^{4}$ Evidently former decay mode leads to opposite sign dileptons (OSDL), also suppressed by $\left|V_{l N}\right|^{4}$, but slightly larger compare to tri-lepton signal. However, significant irreducable backgrounds can come from $t \bar{t}, V V$ (with $V=W, Z)$, as well as $Z+J$ ets after vetoing dilepton invariant mass at Z-pole. Hence we are not considering the OSDL as a signal. Estimate of these backgrounds for OSDL can be found in 69]. Note that their more specific selection criteria are not applicable for our present signal. Similarly, OSDL through VBF is suppressed by $\left|V_{l N}\right|^{4}$ and is beset with large background coming from $W W, \tau \tau$ and $Z Z$ production at VBF [57].
} 
tri-lepton signal via leptonic decays and we have considered their contributions in our simulation. However since we will apply Z-veto (to minimize the SM background), the contribution coming from $Z \nu$ decay mode will be suppressed after final event selection.

As lepton Yukawa is small, the $H \nu$ mode is also not going to contribute to our signal even for higher values of $M_{N}$.

\section{SIMULATION AND RESULTS}

We have implemented the model in FeynRules [70] and generated the Feynman rules compatible with MadGraph5 [11]. After generating Les Houches Event (LHE) [72] file from MadGraph, we have passed that to PYTHIA6 [73] for showering and hadronization.

\subsection{Selection criteria}

To get enhancement in signal over background, we use the following selection criteria [74, 75]:

$\diamond$ Identification criteria of a lepton: pseudorapidity $\left|\eta_{\ell}\right|<2.5$ and $p_{T \ell}>20 \mathrm{GeV}$ have been used.

$\diamond$ Detector efficiency for leptons [76, 77]:

- For electron (either $e^{-}$or $e^{+}$) detector efficiency is $0.7(70 \%)$;

- For muon (either $\mu^{-}$or $\mu^{+}$) detector efficiency is $0.9(90 \%)$.

$\diamond$ Smearing ${ }^{5}$ of electron energy and muon $p_{T}$ are incorporated.

$\diamond$ Lepton-lepton separation: for this $\Delta R_{l l} \geq 0.2$ is used $^{6}$ (due to detector resolution of leptons).

$\diamond$ Lepton-photon separation: this is taken as $\Delta R_{l \gamma} \geq 0.2$ with all the photons having $p_{T_{\gamma}}>10$ $\mathrm{GeV}$.

$\diamond$ Lepton-jet separation: The separation of a lepton with all the jets is set at $\Delta R_{l j} \geq 0.4$; otherwise that lepton is not counted as lepton. Jets are constructed from hadrons using PYCELL within the PYTHIA.

\footnotetext{
${ }^{5}$ Choice of corresponding $\eta$ dependent parameters is similar to one of our earlier work [4]].

${ }^{6}$ Here $\Delta R_{i j}=\sqrt{\left(\eta_{i}-\eta_{j}\right)^{2}-\left(\phi_{i}-\phi_{j}\right)^{2}}$ quantifies the separation between particles $i$ and $j$ in the pseudorapidity $(\eta)$ $\operatorname{azimuth}(\phi)$ plane.
} 
$\diamond$ Hadronic activity cut: This cut is applied to take only pure kind of leptons that have very less hadronic activity around them. The hadronic activity within the cone of radius 0.2 around the lepton should be small, $\frac{\sum p_{T_{\text {hadron }}}}{p_{T_{l}}} \leq 0.2$.

$\diamond$ Hard $p_{T}$ cuts used are: $p_{T l_{1}}>30 \mathrm{GeV}, p_{T l_{2}}>30 \mathrm{GeV}$ and $p_{T l_{3}}>20 \mathrm{GeV}$.

$\diamond$ Missing $p_{T}$ cut: Due to the presence of neutrino, a missing $p_{T}$ cut $(>30 \mathrm{GeV})$ is applied.

$\diamond \mathrm{Z}_{\text {-veto }}{ }^{7}$ is applied to suppress the SM background.

$\diamond \mathrm{VBF}$ cuts $[55,78]$ :

- Central jet veto is also applied, in which we consider any jet with $E_{T 3}>20 \mathrm{GeV}$ and compute the rapidity with respect to the average of the two forward jets: $\eta_{0}=$ $\eta_{3}-\left(\eta_{1}+\eta_{2}\right) / 2$. We veto the event if $\left|\eta_{0}\right|<2$. Central jet veto is applied to suppress the QCD background substantially.

- Charged leptons need to fall in between the rapidities of two forward tagging jets i.e. $\eta_{j, \min }<\eta_{\ell}<\eta_{j, \max }$.

- $p_{T}$ of jets: $p_{T j_{1}, j_{2}}>20 \mathrm{GeV}$.

- Invariant mass of jets: $M_{j_{1} j_{2}}>600 \mathrm{GeV}$.

- Pseudorapidity of jets: $\eta_{j_{1}} \cdot \eta_{j_{2}}<0$ and $\left|\eta_{j_{1}}-\eta_{j_{2}}\right|>4$. Demanding both the tagged jets in opposite hemisphere and a large rapidity separation among them significantly reduces the $\mathrm{BG}$ for $\mathrm{VBF}$.

\subsection{Background}

\subsubsection{For s-channel signal}

To calculate the SM background we consider all channels that can produce or mimic the trilepton production with missing $P_{T}$. We closely follow the reference [74, 79] where similar background analysis was done with the event selection criteria listed as above except the cuts related to the VBF. Events are generated using ALPGEN [80] for the processes coming from $t \bar{t}, t \bar{t}\left(Z / \gamma^{*}\right)$, $t \bar{t} W^{ \pm}, W^{ \pm}\left(Z / \gamma^{*}\right),\left(Z / \gamma^{*}\right)\left(Z / \gamma^{*}\right)$ at the parton level and passed into PYTHIA. As expected $t \bar{t}$ and

\footnotetext{
${ }^{7}$ Same flavored but opposite sign lepton pair invariant mass $m_{\ell_{1} \ell_{2}}$ must be sufficiently away from $Z$ mass, such that, typically, $\left|m_{\ell_{1} \ell_{2}}-M_{Z}\right| \geq 6 \Gamma_{Z} \sim 15 \mathrm{GeV}$.
} 


\begin{tabular}{|c|c|cccr|}
\hline \multirow{2}{*}{ Process } & \multicolumn{5}{|c|}{ Cross section $(f b)$} \\
\cline { 2 - 6 } & $\ell \ell \ell$ & $e e e$ & $e e \mu$ & $e \mu \mu$ & $\mu \mu \mu$ \\
\hline$t \bar{t}$ & 18.972 & 1.1383 & 7.0831 & 8.2214 & 2.5297 \\
\hline$W^{ \pm}\left(Z / \gamma^{*}\right)$ & 10.832 & 0.0677 & 0.1311 & 5.9891 & 4.6440 \\
\hline$\left(Z / \gamma^{*}\right)\left(Z / \gamma^{*}\right)$ & 1.175 & 0.0734 & 0.0525 & 0.6400 & 0.4090 \\
\hline$t \bar{t}\left(Z / \gamma^{*}\right)$ & 1.103 & 0.0429 & 0.1329 & 0.4997 & 0.4275 \\
\hline$t \bar{t} W^{ \pm}$ & 0.639 & 0.0328 & 0.2655 & 0.2424 & 0.0983 \\
\hline TOTAL & $\mathbf{3 2 . 7 2 1}$ & $\mathbf{1 . 3 5 5 1}$ & $\mathbf{7 . 6 6 5 1}$ & $\mathbf{1 5 . 5 9 2 6}$ & $\mathbf{8 . 1 0 8 5}$ \\
\hline
\end{tabular}

TABLE II: Dominant Standard Model background cross sections contributing to tri-lepton and missing transverse energy. These are calculated satisfying all the cuts (except VBF cuts) for the 14 TeV LHC. For each process we also classify the tri-lepton background into four different flavor combinations and present the cross section in each case along with the total contribution.

$W^{ \pm}\left(Z / \gamma^{*}\right)$ contribute dominantly. These and other SM backgrounds are listed in Table II. For each process we classify the tri-lepton signals into four different flavor combinations and compute the cross section in each case along with the total contribution.

\subsubsection{For VBF signal}

Tri-lepton signal with missing $P_{T}$ and two forward jets in VBF can be faked by different SM backgrounds. Processes like $t \bar{t}$ would produce b-jets and mostly effective in central region. Vetoing on jet activities in central region can eliminate most of the non-VBF type SM processes. However most important irreducible background comes from $W^{ \pm} Z$ and $Z Z$ together with two extra forward jets once the gauge bosons decay leptonically. These processes can construct dominant SM background for the VBF production of $3 \ell+\mathbb{E}_{T}$ since they includes the typical VBF topology and hence can easily pass the central jet veto criteria. These backgrounds are calculated ${ }^{8}$ using MadGraph5 and PYTHIA6. In the Table III the dominant background cross sections after satisfying all the cuts including VBF cuts at $14 \mathrm{TeV}$ LHC is tabulated. Like the case of s-channel backgrounds, for each process we also classify the tri-lepton signals into four different flavor combinations and compute the cross section in each case as well as the total contribution.

\footnotetext{
${ }^{8}$ Next to leading order QCD corrections are available in [66, 67].
} 


\begin{tabular}{|c|c|cccc|}
\hline \multirow{2}{*}{ Process } & \multicolumn{5}{|c|}{ Cross section $(f b)$} \\
\cline { 2 - 6 } & $\ell \ell \ell$ & eee & $e e \mu$ & $e \mu \mu$ & $\mu \mu \mu$ \\
\hline$W^{+} Z j j$ & 0.04068 & 0.00073 & 0.00105 & 0.02157 & 0.01734 \\
\hline$W^{-} Z j j$ & 0.01923 & 0.00038 & 0.00055 & 0.00994 & 0.00836 \\
\hline$Z Z j j$ & 0.00094 & 0.00002 & 0.00002 & 0.00066 & 0.00024 \\
\hline TOTAL & $\mathbf{0 . 0 6 0 8 5}$ & $\mathbf{0 . 0 0 1 1 3}$ & $\mathbf{0 . 0 0 1 6 2}$ & $\mathbf{0 . 0 3 2 1 6}$ & $\mathbf{0 . 0 2 5 9 4}$ \\
\hline
\end{tabular}

TABLE III: Dominant Standard Model background cross section contributing to tri-lepton and missing transverse energy associated with two forward jets. These are calculated satisfying all the cuts including VBF cuts for the $14 \mathrm{TeV}$ LHC. Cross sections of four different flavor combinations as well as the total cross section are listed.

\subsection{Signal}

Earlier in section. 3 we have presented the total heavy neutrino production cross sections for different light neutrino hierarchy with basic selection criteria. The crossection for NH scenario was found to be much lower than the IH scenario for $s$-channel. The brancing ratios for decays of $\mathrm{N}$ to final states with $\mu$ and $e$ are also very small for NH. Therefore we will concentrate only on IH scenario henceforth. For this we consider both s-channel and VBF process. Although the VBF cross-section for $\mathrm{IH}$ is lower or comparebale to s-channel cross-section for $\mathrm{NH}$ for lower values of $M_{N}$, the background for VBF procceses are much smaller. Hence we study this channel also for IH. In this section we consider all leptonic decay modes of heavy neutrinos for a benchmark mass of $M_{N}$ at $100 \mathrm{GeV}$ with the cuts discussed in section. 4.1.

\subsubsection{Signal for s-channel}

The signal coming from decay of heavy neutrinos

$$
p p \rightarrow \ell^{ \pm} N \rightarrow \ell^{ \pm}\left(\ell^{\mp} W^{ \pm}\right) \rightarrow \ell^{ \pm} \ell^{\mp} \ell^{ \pm}+\mathbb{E}_{T}, \quad \text { where } \ell \equiv e, \mu .
$$

Table IV lists the final tri-lepton signal cross section through s-channel heavy neutrino production at $14 \mathrm{TeV}$ LHC for the benchmark point $M_{N}=100 \mathrm{GeV}$ incorporating all event selection criteria except VBF cuts as described earlier. The total contribution from the light leptons as well as the contributions from the four different flavor combinations are presented.

As we can see from the Table IV cross section in terms of flavors has the ordering: ee $\mu>$ eee > $e \mu \mu>\mu \mu \mu$. We can understand this in the following way. There are total 8 possibilities which 


\begin{tabular}{|c|c|cccc|}
\hline \multirow{2}{*}{ Hierarchy } & \multicolumn{5}{|c|}{ Cross section $(f b)$} \\
\cline { 2 - 6 } & $\ell \ell \ell$ & eee & $e e \mu$ & $e \mu \mu$ & $\mu \mu \mu$ \\
\hline $\mathrm{IH}$ & 27.07 & 10.297 & 16.314 & 0.459 & 0.0 \\
\hline
\end{tabular}

TABLE IV: Cross section for IH case. Final tri-lepton signal cross section through s-channel heavy neutrino production at the $14 \mathrm{TeV}$ LHC for the benchmark point $M_{N}=100 \mathrm{GeV}$ including all event selection cuts except VBF cuts. We classify the tri-lepton signals into four different flavor combinations and present the cross section in each case along with the total light lepton contribution.

can produce $\ell \ell \ell$ events. There is only one way to produce $\mu \mu \mu$ and eee final states. However, there are three possible ways to get the $e e \mu$ channel depending on which one of $\ell_{i}$ 's in figure 2 is associated with $e$ and $\mu$. Similarly for the $e \mu \mu$ final state also we get 3 possibilities. The amplitude for eee channel $\sim V_{e N}^{4}$; the ee $\mu$ channel goes as $\sim V_{e N}^{2}+2 V_{e N} V_{\mu N}$; the $e \mu \mu$ channel goes as $\sim V_{\mu N}^{2}+2 V_{e N} V_{\mu N}$ while the $\mu \mu \mu$ channel as $\sim V_{\mu N}^{2}$. Since $V_{e N}>>V_{\mu N}$, the eee and ee $\mu$ cross sections are much larger whereas $\mu \mu \mu$ cross section is negligible. ee $\mu$ crossection is higher than the eee crossection because of higher muon efficiency in the detector, whereas the small $e \mu \mu$ crossection is due to a very tiny value of $V_{\mu N}$.

One can also compute the ratios of events with different flavour compositions in which some of the common systematic uncertainties can get cancelled. For example ee $\mu /$ eee $\sim \epsilon$ where $\epsilon$ denotes the relative efficiency of detection of muon over electron, ee $\mu / \mu \mu \mu \sim \epsilon V_{e N}^{4} / V_{\mu N}^{4} ;$ eee $/ e \mu \mu \sim$ $\epsilon^{2} V_{e N}^{4} / V_{\mu N}^{4}$ etc. Since for a fixed $y_{\nu}$, which in turn implies specific values for phases, the variation of the light-heavy mixing angles are not very much with oscillation parameters, these ratios vary within a very narrow range ${ }^{9}$ and hence can be used to test the model. Ofcourse for different phase choices a different $y_{\nu}$ and hence different predictions can be obtained. However, a smaller value in $y_{\nu}$ would result in a lower event rate and hence it would be difficult to test at the LHC.

\subsubsection{Signal for $V B F$}

In this section we present the results for the case where $N$ is produced by VBF:

$$
p p \rightarrow \ell^{ \pm} N j j \rightarrow \ell^{ \pm}\left(\ell^{\mp} W^{ \pm}\right) j j \rightarrow \ell^{ \pm} \ell^{\mp} \ell^{ \pm}+\mathbb{E}_{T}+j j(\text { forward jets }), \quad \text { where } \ell \equiv e, \mu .
$$

\footnotetext{
${ }^{9}$ Note that, the allowed magnitude of mixings are as following: For a fixed value of $y_{\nu}(=0.4), \alpha(=3 \pi / 4), \delta(=0)$ and $M_{N}(=100) \mathrm{GeV}$, the magnitude of $\left|V_{e N}\right|$ and $\left|V_{\mu N}\right|$ vary in a very small range for $3 \sigma$ variation of oscillation parameters; $\left|V_{e N}\right|=0.471-0.484,\left|V_{\mu N}\right|=1.236 \times 10^{-4}-1.272 \times 10^{-4}$. However, $\left|V_{\tau N}\right|$ varies little higher; $\left|V_{\tau N}\right|=0.092-0.147$. Since we are considering modes involving only $e$ and $\mu$, the crossections are likely to vary by a small amount for different set of oscillation parameters.
} 


\begin{tabular}{|c|c|cccc|}
\hline \multirow{2}{*}{ Hierarchy } & \multicolumn{5}{|c|}{ Cross section $(f b)$} \\
\cline { 2 - 6 } & $\ell \ell \ell$ & eee & $e e \mu$ & $e \mu \mu$ & $\mu \mu \mu$ \\
\hline $\mathrm{IH}$ & 0.018068 & $7.09 \times 10^{-3}$ & $1.06 \times 10^{-2}$ & $4.06 \times 10^{-4}$ & 0.00 \\
\hline
\end{tabular}

TABLE V: Final tri-lepton Signal through VBF production of heavy neutrino for the benchmark point $M_{N}=100 \mathrm{GeV}$ at $14 \mathrm{TeV} \mathrm{LHC}$ for IH after all event selection cuts.
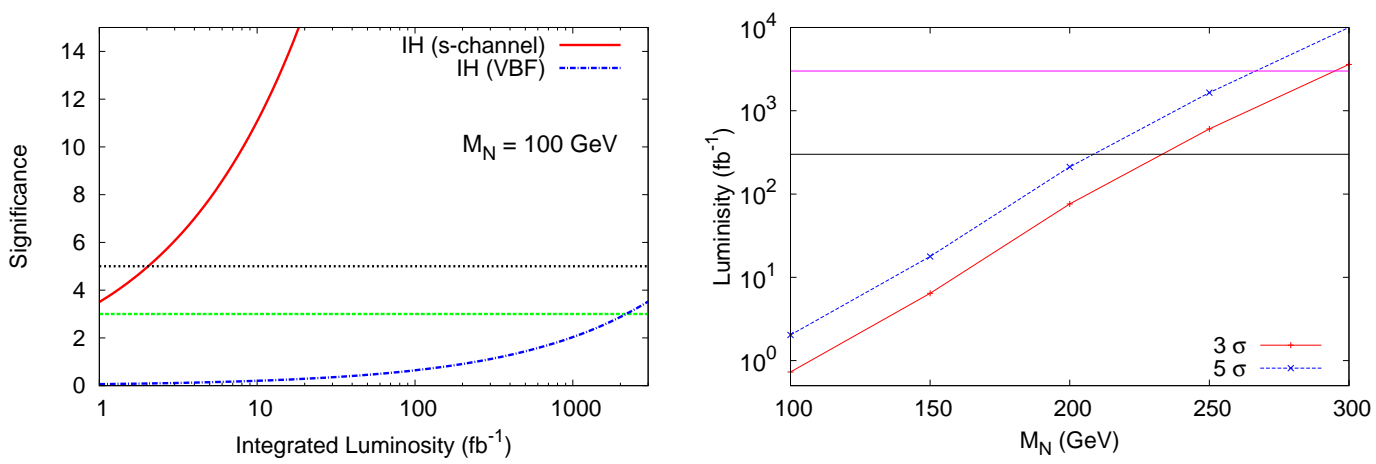

FIG. 6: (Left) The variation of significance $S / \sqrt{S+B}$ for the s-channel production signal for benchmark point $M_{N}=100 \mathrm{GeV}$ with the integrated luminosity available for the low luminosity option at $14 \mathrm{TeV}$ LHC. Black-dotted (green-dashed) line parallel to the x-axis represents $5 \sigma(3 \sigma)$ significance. (Right) The lines for $3 \sigma$ (red) and $5 \sigma$ (blue) significance in terms of heavy neutrino mass and integrated luminosity. With $300 \mathrm{fb}^{-1}$ luminosity at LHC14 the heavy neutrino mass in this model can be probed up to $\sim 210(230) \mathrm{GeV}$ with $\sim 5 \sigma$ $(3 \sigma)$ significance. For very high luminosity of $3000 \mathrm{fb}^{-1}$ this can reach up to $\sim 270(295) \mathrm{GeV}$.

In Table $\square$ we present the final tri-lepton signal cross sections through VBF production of heavy neutrinos at the $14 \mathrm{TeV}$ LHC for the benchmark point $M_{N}=100 \mathrm{GeV}$, after including all cuts. Here we have only shown the case of inverted hierarchy and signal is found to be quite small. Although VBF backgrounds are small, the tiny production cross sections are insufficient for giving any signal with integrated luminosity of $300 \mathrm{fb}^{-1}$. Some indications from VBF can appear only at the HL-LHC $\left(3000 \mathrm{fb}^{-1}\right)$. However, $5 \sigma$ significance can not be reached even for $M_{N}=100 \mathrm{GeV}$.

\section{DISCOVERY POTENTIAL}

After numerical computation of all necessary signals and backgrounds, results are better represented in terms of significance, defined as $S / \sqrt{S+B}$, where $S(B)=\mathcal{L} \sigma_{S(B)}$. Here $\mathcal{L}$ being integrated luminosity available for the collider at certain machine energy and $\sigma_{S(B)}$ is the final cross section after all event selection, for given parameters like heavy neutrino mass and corresponding allowed couplings. Fig. 6 (Left) demonstrates the expected significance coming from 
s-channel production of heavy Dirac neutrino of mass $100 \mathrm{GeV}$ as a function of integrated luminosity at $14 \mathrm{TeV}$ LHC. In the figure black-dotted (green-dashed) line shows $5 \sigma(3 \sigma)$ significance. From the figure it is clear that for the case of s-channel signal in the IH scenario, $3 \sigma(5 \sigma)$ significance can be achieved within the integrated luminosity $\sim 0.73(2.03) \mathrm{fb}^{-1}$. In the case of VBF channel $3 \sigma$ significance can be achieved with $2175 \mathrm{fb}^{-1}$ luminosity, while $5 \sigma$ significance is not achievable within $3000 \mathrm{fb}^{-1}$ luminosity which is planned for the HL-LHC.

Fig. 6 (Right) shows the lines for $3 \sigma$ (red) and $5 \sigma$ (blue) significance in terms of heavy neutrino mass and integrated luminosity. With $300 \mathrm{fb}^{-1}$ luminosity at LHC14 the heavy neutrino mass in this model can be probed up to $\sim 210(230) \mathrm{GeV}$ with $\sim 5 \sigma(3 \sigma)$ significance. For very high luminosity of $3000 \mathrm{fb}^{-1}$ this can reach up to $\sim 270(295) \mathrm{GeV}$. For VBF signal, since $M_{N}=100$ $\mathrm{GeV}$ itself requires a very large integrated luminosity; higher values of $M_{N}$ are not possible to explore.

\section{SUMMARY AND CONCLUSION}

In this work we have considered $\mathrm{TeV}$ scale minimal linear seesaw model which generates correct order of light neutrino masses and has sizable light-heavy mixing to produce heavy neutrinos at colliders like LHC. One of the important features of this model is that it can be fully reconstructible from oscillation data excepting an overall factor $y_{\nu}$ characterizing the Dirac Yukawa matrix. However this parameter gets constrained by LFV and vacuum meta-stability bounds. The neutral fermion mass spectrum of this model consists of one massless, two light and two heavy neutrinos.

We have studied the collider phenomenology of TeV scale linear seesaw at $14 \mathrm{TeV}$ LHC. The heavy neutrinos in this model can be dominantly produced through the s-channel. In a leading order calculation, subsequent decay of these leads to characteristic tri-lepton signal with missing $p_{T}$. We also consider the production of heavy neutrinos through the VBF process. The signal for this is tri-leptons with additional two forward jets which can be tagged. Both these signals as well as SM backgrounds have been estimated with realistic simulations using MadGraph and PYTHIA.

We found that s-channel tri-lepton production process have potential to be discovered at the LHC for IH scenario. However due to severe constraint on the light-heavy mixing coming form LFV in the case of NH scenario, both $s$-channel and VBF can not be probed at the $14 \mathrm{TeV}$ LHC with proposed luminosity. For a benchmark point with a heavy neutrino mass $M_{N}=100 \mathrm{GeV}, 3 \sigma$ significance can be achieved with integrated luminosity of $\sim 0.73(2175) \mathrm{fb}^{-1}$ for $s$-channel(VBF) signal in the IH scenario. $5 \sigma$ significance can be reached for $s$-channel signal with a integrated 
luminosity of $\sim 2 \mathrm{fb}^{-1}$, however for VBF signal the required luminosity is $\sim 6042 f b^{-1}$, which is beyond the reach of projected luminosity at the LHC. Discovery reach in the tri-lepton channel can be achieved upto the heavy neutrino mass of $\sim 210(230) \mathrm{GeV}$ with $\sim 5 \sigma(3 \sigma)$ significance at the low luminosity $\left(300 \mathrm{fb}^{-1}\right)$ option of $14 \mathrm{TeV}$ LHC. In the high luminosity $\left(3000 \mathrm{fb}^{-1}\right)$ search, reach is upto $\sim 270(295) \mathrm{GeV}$. Whereas, VBF channel can only reach upto $\sim 3 \sigma$ for $M_{N}$ at $100 \mathrm{GeV}$. Our analysis uses values for the elements, $V_{l N}$ of the light-heavy mixing matrix, which are consistent with the constraints coming from vacuum metastability and LFV. Any freedom of choosing larger values (e.g. $\sim \mathcal{O}(1))$ for these parameters can extend the discovery limit by a very significant amount. With the constraints used in this work, for $V_{l N}$, a detectable tri-lepton signal can only be obtained for the inverted hierarchical scenario with particular choices of phases leading to large $y_{\nu}$. One can also compute the ratios of events with different flavour compositions which are proportional to the elements $V_{l N}$. They vary only within a narrow range with the $3 \sigma$ variations of oscillation parameters and thus the model has very definite predicions for these ratios.

[1] G. Aad et al. (ATLAS Collaboration), Phys.Lett. B716, 1 (2012), arXiv:1207.7214 [hep-ex]

[2] S. Chatrchyan et al. (CMS Collaboration), Phys.Lett. B716, 30 (2012), arXiv:1207.7235 [hep-ex]

[3] P. Ade et al. (Planck Collaboration)(2013), arXiv:1303.5076 [astro-ph.CO]

[4] S. Weinberg, Phys.Rev.Lett. 43, 1566 (1979)

[5] P. Minkowski, Phys.Lett. B67, 421 (1977)

[6] T. Yanagida, Conf.Proc. C7902131, 95 (1979)

[7] M. Gell-Mann, P. Ramond, and R. Slansky, Conf.Proc. C790927, 315 (1979)

[8] S. Glashow, NATO Adv.Study Inst.Ser.B Phys. 59, 687 (1980)

[9] R. N. Mohapatra and G. Senjanovic, Phys.Rev.Lett. 44, 912 (1980)

[10] A. Pilaftsis, Z.Phys. C55, 275 (1992), arXiv:hep-ph/9901206 [hep-ph]

[11] J. Gluza, Acta Phys.Polon. B33, 1735 (2002), arXiv:hep-ph/0201002 [hep-ph]

[12] A. Pilaftsis, Phys.Rev.Lett. 95, 081602 (2005), arXiv:hep-ph/0408103 [hep-ph]

[13] J. Kersten and A. Y. Smirnov, Phys.Rev. D76, 073005 (2007), arXiv:0705.3221 [hep-ph]

[14] Z.-z. Xing, Prog.Theor.Phys.Suppl. 180, 112 (2009), arXiv:0905.3903 [hep-ph]

[15] X.-G. He, S. Oh, J. Tandean, and C.-C. Wen, Phys.Rev. D80, 073012 (2009), arXiv:0907.1607 [hep-ph]

[16] A. Ibarra, E. Molinaro, and S. Petcov, JHEP 1009, 108 (2010), arXiv:1007.2378 [hep-ph]

[17] F. F. Deppisch and A. Pilaftsis, Phys.Rev. D83, 076007 (2011), arXiv:1012.1834 [hep-ph]

[18] R. Adhikari and A. Raychaudhuri, Phys.Rev. D84, 033002 (2011), arXiv:1004.5111 [hep-ph]

[19] I. Gogoladze, N. Okada, and Q. Shafi, Phys.Lett. B672, 235 (2009), arXiv:0809.0703 [hep-ph]

[20] K. Babu, S. Nandi, and Z. Tavartkiladze, Phys.Rev. D80, 071702 (2009), arXiv:0905.2710 [hep-ph] 
[21] F. Bonnet, D. Hernandez, T. Ota, and W. Winter, JHEP 0910, 076 (2009), arXiv:0907.3143 [hep-ph]

[22] S. Kanemura and T. Ota, Phys.Lett. B694, 233 (2010), arXiv:1009.3845 [hep-ph]

[23] Y. Liao, G.-Z. Ning, and L. Ren, Phys.Rev. D82, 113003 (2010), arXiv:1008.0117 [hep-ph]

[24] I. Picek and B. Radovcic, Phys.Lett. B687, 338 (2010), arXiv:0911.1374 [hep-ph]

[25] Y. Liao, JHEP 1106, 098 (2011), arXiv:1011.3633 [hep-ph]

[26] K. Kumericki, I. Picek, and B. Radovcic, Phys.Rev. D86, 013006 (2012), arXiv:1204.6599 [hep-ph]

[27] K. L. McDonald, JHEP 1307, 020 (2013), arXiv:1303.4573 [hep-ph]

[28] A. Zee, Phys.Lett. B93, 389 (1980)

[29] A. Zee, Nucl.Phys. B264, 99 (1986)

[30] K. Babu, Phys.Lett. B203, 132 (1988)

[31] L. M. Krauss, S. Nasri, and M. Trodden, Phys.Rev. D67, 085002 (2003), arXiv:hep-ph/0210389 [hep-ph]

[32] E. Ma, Phys.Rev. D73, 077301 (2006), arXiv:hep-ph/0601225 [hep-ph]

[33] M. Aoki, S. Kanemura, and O. Seto, Phys.Rev.Lett. 102, 051805 (2009), arXiv:0807.0361 [hep-ph]

[34] P. B. Dev and A. Pilaftsis, Phys.Rev. D86, 113001 (2012), arXiv:1209.4051 [hep-ph]

[35] M. Gustafsson, J. M. No, and M. A. Rivera(2014), arXiv:1402.0515 [hep-ph]

[36] R. Mohapatra and J. Valle, Phys.Rev. D34, 1642 (1986)

[37] W.-Y. Keung and G. Senjanovic, Phys.Rev.Lett. 50, 1427 (1983)

[38] A. Datta, M. Guchait, and A. Pilaftsis, Phys.Rev. D50, 3195 (1994), arXiv:hep-ph/9311257 [hep-ph]

[39] T. Han and B. Zhang, Phys.Rev.Lett. 97, 171804 (2006), arXiv:hep-ph/0604064 [hep-ph]

[40] S. Bray, J. S. Lee, and A. Pilaftsis, Nucl.Phys. B786, 95 (2007), arXiv:hep-ph/0702294 [HEP-PH]

[41] F. del Aguila, J. Aguilar-Saavedra, and R. Pittau, JHEP 0710, 047 (2007), arXiv:hep-ph/0703261 [hep-ph]

[42] F. del Aguila and J. Aguilar-Saavedra, Nucl.Phys. B813, 22 (2009), arXiv:0808.2468 [hep-ph]

[43] A. Atre, T. Han, S. Pascoli, and B. Zhang, JHEP 0905, 030 (2009), arXiv:0901.3589 [hep-ph]

[44] S. Chatrchyan et al. (CMS Collaboration), Phys.Lett. B717, 109 (2012), arXiv:1207.6079 [hep-ex]

[45] P. S. B. Dev, A. Pilaftsis, and U.-k. Yang, Phys.Rev.Lett. 112, 081801 (2014), arXiv:1308.2209 [hep-ph]

[46] A. Das, P. Bhupal Dev, and N. Okada(2014), arXiv:1405.0177 [hep-ph]

[47] F. del Aguila and J. Aguilar-Saavedra, Phys.Lett. B672, 158

[48] C.-Y. Chen and P. B. Dev, Phys.Rev. D85, 093018 (2012), arXiv:1112.6419 [hep-ph]

[49] A. Das and N. Okada(2012), arXiv:1207.3734 [hep-ph]

[50] O. Eboli, J. Gonzalez-Fraile, and M. Gonzalez-Garcia, JHEP 1112, 009 (2011), arXiv:1108.0661 [hep-ph]

[51] S. Chatrchyan et al. (CMS Collaboration)(2014), arXiv:1404.5801 [hep-ex]

[52] M. Gavela, T. Hambye, D. Hernandez, and P. Hernandez, JHEP 0909, 038 (2009), arXiv:0906.1461 [hep-ph]

[53] S. Khan, S. Goswami, and S. Roy, Phys.Rev. D89, 073021 (2014), arXiv:1212.3694 [hep-ph]

[54] M. Malinsky, T. Ohlsson, Z.-z. Xing, and H. Zhang, Phys.Lett. B679, 242 (2009), 
arXiv:0905.2889 [hep-ph]

[55] D. L. Rainwater(1999), arXiv:hep-ph/9908378 [hep-ph]

[56] A. Datta, P. Konar, and B. Mukhopadhyaya, Phys.Rev.Lett. 88, 181802 (2002), arXiv:hep-ph/0111012 [hep-ph]

[57] D. Choudhury, A. Datta, K. Huitu, P. Konar, S. Moretti, et al., Phys.Rev. D68, 075007 (2003), arXiv:hep-ph/0304192 [hep-ph]

[58] G.-C. Cho, K. Hagiwara, J. Kanzaki, T. Plehn, D. Rainwater, et al., Phys.Rev. D73, 054002 (2006), arXiv:hep-ph/0601063 [hep-ph]

[59] P.-H. Gu and U. Sarkar, Phys.Lett. B694, 226 (2010), arXiv:1007.2323 [hep-ph]

[60] H. Zhang and S. Zhou, Phys.Lett. B685, 297 (2010), arXiv:0912.2661 [hep-ph]

[61] M. Hirsch, S. Morisi, and J. Valle, Phys.Lett. B679, 454 (2009), arXiv:0905.3056 [hep-ph]

[62] F. Bazzocchi, Phys.Rev. D83, 093009 (2011), arXiv:1011.6299 [hep-ph]

[63] W. Grimus and L. Lavoura, JHEP 0011, 042 (2000), arXiv:hep-ph/0008179 [hep-ph]

[64] S. Khan, Nucl.Phys. B864, 38 (2012), arXiv:1203.5043 [hep-ph]

[65] J. de Blas, EPJ Web Conf. 60, 19008 (2013), arXiv:1307.6173 [hep-ph]

[66] G. Bozzi, B. Jager, C. Oleari, and D. Zeppenfeld, Phys.Rev. D75, 073004 (2007), arXiv:hep-ph/0701105 [hep-ph]

[67] B. Jager, C. Oleari, and D. Zeppenfeld, Phys.Rev. D73, 113006 (2006), arXiv:hep-ph/0604200 [hep-ph]

[68] J. Pumplin, D. Stump, J. Huston, H. Lai, P. M. Nadolsky, et al., JHEP 0207, 012 (2002), arXiv:hep-ph/0201195 [hep-ph]

[69] J. Eckel, M. J. Ramsey-Musolf, W. Shepherd, and S. Su, JHEP 1411, 117 (2014), arXiv:1408.2841 [hep-ph]

[70] N. D. Christensen and C. Duhr, Comput.Phys.Commun. 180, 1614 (2009), arXiv:0806.4194 [hep-ph]

[71] J. Alwall, M. Herquet, F. Maltoni, O. Mattelaer, and T. Stelzer, JHEP 1106, 128 (2011), arXiv:1106.0522 [hep-ph]

[72] J. Alwall, A. Ballestrero, P. Bartalini, S. Belov, E. Boos, et al., Comput.Phys.Commun. 176, 300 (2007), arXiv:hep-ph/0609017 [hep-ph]

[73] T. Sjostrand, S. Mrenna, and P. Z. Skands, JHEP 0605, 026 (2006), arXiv:hep-ph/0603175 [hep-ph]

[74] G. Bambhaniya, J. Chakrabortty, S. Goswami, and P. Konar, Phys.Rev. D88, 075006 (2013), arXiv:1305.2795 [hep-ph]

[75] B. Mukhopadhyaya and S. Mukhopadhyay, Phys.Rev. D82, 031501 (2010), arXiv:1005.3051 [hep-ph]

[76] G. Aad et al. (ATLAS Collaboration)(2009), arXiv:0901.0512 [hep-ex]

[77] G. Bayatian et al. (CMS Collaboration), J.Phys. G34, 995 (2007)

[78] E. Yazgan, J. Damgov, N. Akchurin, V. Genchev, D. Green, et al., Eur.Phys.J. C53, 329 (2008), arXiv:0706.1898 [hep-ex]

[79] G. Bambhaniya, J. Chakrabortty, J. Gluza, M. Kordiaczynska, and R. Szafron, JHEP 1405, 033 (2014), arXiv:1311.4144 [hep-ph] 
[80] M. L. Mangano, M. Moretti, F. Piccinini, R. Pittau, and A. D. Polosa, JHEP 0307, 001 (2003), arXiv:hep-ph/0206293 [hep-ph] 